\title{
ON CERTAIN DETERMINANTS CONNECTED WITH A PROBLEM IN CELESTIAL MECHANICS.
}

BY MR. H. E. BUCHANAN.

(Read before the Chicago Section of the American Mathematical Society, January 2, 1909.)

1. The solutions of the $n$-body problem, in which all are in the same straight line and moving in circles, are defined by the the following set of equations : *

$$
\begin{gathered}
0+\frac{m_{2}}{r_{12}^{2}}+\frac{m_{3}}{r_{13}^{2}}+\cdots+\frac{m_{n}}{r_{1 n}^{2}}=-\omega^{2} x_{1}, \\
-\frac{m_{1}}{r_{12}^{2}}+0+\frac{m_{3}}{r_{23}^{2}}+\cdots+\frac{m_{n}}{r_{2 n}^{2}}=-\omega^{2} x_{2}, \\
-\frac{m_{1}}{r_{13}^{2}}-\frac{m_{n}}{r_{23}^{2}}+0+\cdots+\frac{m_{n}}{r_{3 n}^{2}}=-\omega^{2} x_{3}, \\
\vdots \\
-\frac{m_{1}}{r_{1 n}^{2}}-\frac{m_{2}}{r_{2 n}^{2}}-\quad \cdots+0=-\omega^{2} x_{n},
\end{gathered}
$$

where the $m_{i}$ stand for the masses and the $x_{i}$ are their respective distances from the center of gravity of the system.

In his lectures Professor Moulton has considered these equations in two ways :

(1) Given the masses to find the distances.

(2) Given the distances to find the masses.

This paper is concerned with the second problem and its purpose is to answer two questions which he raised.

2. In (2) there is an unique determination of the masses if $J$, the determinant of the left members, never vanishes. $J$ is a skew symmetric determinant, whose properties are quite different according as its order is even or odd. When $n$ is even it is a perfect square, $\uparrow$ and it will be shown that in this case $J$

\footnotetext{
* See Moulton's Celestial Mechanics, p. 213, for the three-body problem. The notation is here chosen so that $x_{1}<x_{2}<\cdots<x^{n}$.

† Cayley, Coll. Papers, 1, p. 412.
} 
never vanishes for any selection of $r_{i j}$. It is only necessary to consider the Pfaffian

$$
\begin{aligned}
& J^{\frac{1}{2}}=\left|\begin{array}{lllll}
\frac{1}{r_{12}^{2}} & \frac{1}{r_{13}^{2}} & \frac{1}{r_{14}^{2}} & \cdots & \frac{1}{r_{1 n}^{2}}
\end{array}\right| \\
& \begin{array}{llll}
\frac{1}{r_{23}^{2}} & \frac{1}{r_{24}^{2}} & \cdots & \frac{1}{r_{2 n}^{2}}
\end{array} \\
& \begin{array}{ccc}
\frac{1}{r_{34}^{2}} & \cdots & \frac{1}{r_{3 n}^{2}} \\
& & \vdots \\
& & \frac{1}{r_{n-1, n}^{2}}
\end{array} \mid
\end{aligned}
$$

Let a definite choice be made for all the $x_{i}$ from $x_{1}$ to $x_{n-1}$ inclusive. Let $x_{n}$ vary between the limits $x_{n-1}$ and $+\infty$. $x_{n}$ can be written $x_{n}=x_{n-1}+x$, where $x$ varies from 0 to $+\infty$. The Pfaffian (2) then becomes a function of $x$ whose properties we propose to study :

$$
P(x)=\left|\begin{array}{cccc}
\frac{1}{r_{12}^{2}} & \frac{1}{r_{13}^{2}} & \cdots & \frac{1}{\left(x_{n-1}+x-x_{1}\right)^{2}} \\
& \frac{1}{r_{23}^{2}} & \cdots & \frac{1}{\left(x_{n-1}+x-x_{2}\right)^{2}} \\
& & & \frac{1}{x^{2}}
\end{array}\right| .
$$

One can verify that $P(x)$ has the following properties :

(a) The terms of the last column are arranged in increasing order, $1 / x^{2}$ being the largest.

(b) Differentiation as to $x$ serves only to multiply $P(x)$ by a constant factor and to increase the power of the denominators in the last column.

(c) $P(x)$, as well as all its derivatives of finite order, remains finite and continuous for $0<x<\infty$.

(d) $P(x)$ and all its derivatives vanish for $x=\infty$.

By Rolle's theorem and $(c),(d)$, a necessary condition that $P(x)$ vanish for $0<x<\infty$ is that $P^{\prime}(x)$ vanish in the same interval. A necessary condition that $P^{\prime}(x)$ vanish is that $P^{\prime \prime}(x)$ vanish, and so on ; a necessary condition that $P^{(n-1)}(x)$ vanish is 
that $P^{(n)}(x)$ vanish. On the other hand if $P^{\prime}(x)$ never vanishes then $P(x)$ does not, and if $P^{\prime \prime}(x)$ never vanishes $P^{\prime}(x)$ does not, finally, if $P^{(n)}(x)$ never vanishes $P^{(n-1)}(x)$ does not. From the properties $(a)$ and $(b)$ it follows that for $n$ sufficiently great and $0<x<\infty, P^{(n)}(x)$ never is zero since the term coming from $1 / x^{2}$ will be greater than the sum of all the other terms unless its coefficient vanishes. This coefficient is the Pfaffian of order $n-2$, and we can apply the same method to it as to $P(x)$, so that it does not vanish unless the minor of order $n-4$ vanishes, etc. But the minor of order 2 does not vanish, therefore by induction none of the minors vanishes and the theorem is proven.

3. When $n$ is odd the determinant of the left members of (1) is identically zero.* Then we consider $\dagger$ the first $n-1$ equations. Transpose the terms containing $m_{n}$ and solve for $m_{1}, \cdots, m_{n-1}$. This is uniquely possible by $\S 2$. Substituting these solutions in the last equation and arranging, there results

$$
F\left(x_{1}, \cdots, x_{n}\right) \omega^{2}+\phi\left(x_{1}, \cdots, x_{n}\right) m_{n}=0 .
$$

$\phi\left(x_{1}, \cdots, x_{n}\right)$ is the determinant of the left members and is identically zero since $n$ is odd. In order that there be a solution at all the following condition must be satisfied

$$
F\left(x_{1}, \cdots, x_{n}\right)=0 .
$$

$m_{n}$ can then be taken arbitrarily and the remaining $m_{j}$ are uniquely determined. Not only so, but it will be shown that after $m_{n}$ and all of the $x$ 's except one has been chosen arbitrarily then that one is uniquely determined by the equation

$$
F\left(x_{1}, \cdots, x_{n}\right)=0 .
$$

4. One easily finds

$$
F=\left|\begin{array}{cccc}
x_{1} & x_{2} & \cdots & x_{n} \\
& \frac{1}{r_{12}^{2}} & \cdots & \frac{1}{r_{1 n}^{2}} \\
& & & \vdots \\
& & & \frac{1}{r_{n-1, n}^{2}}
\end{array}\right|=0 .
$$

* Cayley, Coll. Papers, I, p. 41\%.

$\dagger$ According to Moulton's Lectures. 
Choose $x_{2}, \cdots, x_{n}$ arbitrary constants, while $x_{1}$ varies between the limits $-\infty$ and $x_{2}$. It is easily seen that

$$
F(-\infty)=\infty, \quad F\left(x_{2}\right)=\infty,
$$

the sign being undetermined. Then if the $d F / d x_{1}$ never vanishes $F$ must vanish once and but once in the interval $-\infty<x_{1}<x_{2} . \quad x_{1}$ occurs in the second frame line; therefore on differentiating we have

$$
\frac{d F}{d x_{1}}=\left|\begin{array}{cccc}
1 & x_{2} & \cdots & x_{n} \\
\frac{2}{r_{12}^{3}} & \cdots & \frac{2}{r_{1 n}^{3}} \\
& & & \frac{1}{r_{n-1, n}^{2}}
\end{array}\right| .
$$

Now make a definite choice for $x_{1}$, say $x_{1}^{(0)}$, such that

$$
-\infty<x_{1}^{(0)}<x_{2}
$$

then consider the Pfaffian

$$
P_{1}(\mu)=\left|\begin{array}{cccc}
\frac{1}{2} r_{12}^{3} & & & \\
\left(\frac{1}{2} r_{12}-\mu\right)^{3} & x_{2} & \cdots & x_{n} \\
& \frac{2}{\left(r_{12}-\mu\right)^{3}} & \cdots & \frac{2}{\left(r_{1 n}-\mu\right)^{3}} \\
& & & \vdots \\
& & & \frac{1}{r_{n-1, n}^{2}}
\end{array}\right| .
$$

Let $\mu$ vary from $-\infty$ to $\frac{1}{2}\left(x_{2}-x_{1}^{0}\right)=\frac{1}{2} r_{12}$; then in this interval $P_{1}(\mu)$ has the properties :

(a) The denominators of the second frame line are arranged in increasing order, $\frac{\frac{1}{2} r_{12}^{3}}{\left(\frac{1}{2} r_{12}-\mu\right)^{3}}$ being the smallest.

(b) Differentiation as to $\mu$ multiplies $P_{1}$ by a constant factor and increases the power of these denominators.

$$
P_{1}(0)=\left.\frac{d F}{d x_{1}}\right|^{\mid x=x_{1}^{(0)}},
$$


and $P_{1}$ and all its derivatives vanish for $\mu=-\infty$ but remain finite and continuous for $-\infty<\mu<\frac{1}{2} r_{12}$; and finally $P_{1}\left(\frac{1}{2} r_{12}\right)$ $=\infty$. Reasoning then as in $\S 2$ we conclude that $P_{1}(\mu)$ never vanishes for any value, $-\infty<\mu<\frac{1}{2} r_{12}$; in particular it does not vanish when $\mu$ has the value 0 , that is, by $c$,

$$
\left.\frac{d F}{d x_{1}}\right|^{x=x_{1}^{(0)}} \neq 0 .
$$

Since the above proof does not depend on the value assigned to $x_{1}$ it follows that $d F / d x_{1}$ is never zero for any $-\infty<x_{1}<x_{2}$ and therefore $F$ vanishes once and but once in that interval.

5. In order to complete the theorem one must show that when $x_{i}$ varies continuously between $x_{i-1}$ and $x_{i+1} F$ vanishes uniquely. The course of reasoning is the same as that above and we have to show that $d F^{\prime} / d x_{i}$ never vanishes for $r_{i-1}<x_{i}<x_{i+1} . \quad x_{i}$ occurs in the $i$ th frame line only. Again put $x_{i}=x_{i}^{\left({ }^{(0)}\right.}$ a fixed constant such that $x_{i-1}<x_{i}^{0}<x_{i+1}$ and consider the Pfaffian

$$
\begin{gathered}
P_{i}(\mu)=\left|\begin{array}{cccc}
x_{1} \cdots & q(\mu) & \cdots & x_{n} \\
\cdots & q(\mu) \frac{2}{r_{1 i}^{3}} & \cdots & \frac{1}{r_{i n}^{2}} \\
\vdots & & & \\
q(\mu) \frac{2}{r_{i-1, i}^{3}} & & \frac{1}{r_{i-1, n}^{2}} \\
\frac{2}{\left(r_{i, i+1}-\mu\right)^{3}} & \cdots & \frac{2}{\left(r_{i n}-\mu\right)^{3}}
\end{array}\right| \\
q(\mu)=\frac{1}{2}\left(r^{3}{ }_{i, i+1}\right) /\left[\frac{1}{2}\left(r_{i, i+1}\right)-\mu\right]^{3} .
\end{gathered}
$$

It can be shown that the coefficient of $q(\mu)$ in $P_{i}(\mu)$ does not vanish; then the properties $(a)(b)(c)$ above hold, and the proof is completed as before.

Chicago, Ill.,

May, 1908. 